\title{
Development and Design of Music Education Resource Library Based on Web
}

\author{
Zhe $\mathrm{Li}^{1, \mathrm{a}}$, Hui Ma ${ }^{2, \mathrm{~b}}$ \\ ${ }^{1}$ College of International Exchange, Bohai University, Jinzhou, 121013, China \\ ${ }^{2}$ School of Mathematics and Physics, Bohai University, Jinzhou, 121013, China \\ a503412508@qq.com, bhuima2016@163.com
}

Keywords: music education; Web; resource library; MVC framework; information retrieval

\begin{abstract}
Music as a part of people's leisure and cultural life is rapidly developing and constantly updating, there are many music has gradually been forgotten by people, the development of network technology provides an opportunity for better protecting music education resources; so the study of developing and designing music education resource library based on Web has an important significance. According to the principles of music education resource library design, firstly, this paper studied the requirement analysis in the process of developing and designing music education resource library, mainly using of UML technology to build use case diagrams describe it; secondly, studied the structure of MVC framework used in developing recourse library, elaborated its conception; finally describes the overall design of music education resource library, describes all features that music education resource library can be achieved. Contents of this paper provide a truly effective information retrieval service for the majority of the teaching staff and students and have a role in promoting the development of music.
\end{abstract}

\section{Introduction}

Chinese Music is a very important part of history and culture in five thousand years, its unique musical form and content are also the historical evidence and the spiritual and cultural symbolic for the history and civilization of the Chinese [1]. Music has infinite charm, is an important part of amateur cultural life for people, the rapid development of network technology vigorously promotes the process of music development. For better management, using of the collected music education resources, this paper designed and developed "music education resource library based on Web," using a computer database to store music (including formed audio files and music by playing), using computer to collect, process, store, query and retrieve music education resource, to support to exchange research and the results of music [2]. The development and design of music education resource library provides a solid foundation for inheriting a large number of music education resources, standardized managing and creating more standardized music education resource library.

Compared music education resource library with the general information resource library, not only has a general, but also has a particularity, music education resource library is a subset of the common resource library, so its general refers to having the general attributes of common resource library; particularity refers to some information contained in the music education resource library are more than the information contained in the common resource library. Design and development of music education resource library should contains several principles [3]: (1) Structured and modular, reasonable structure can ensure the usability and scalability for music education resources; (2) digitization and networking, it is conducive to preserve and retrieval information; (3) dynamic, mainly refers to constantly updating and maintaining.

\section{Requirement Analysis}

Requirements analysis phase mainly studies the goal of the system, through communication with users, access to their real requirement, and through modeling to analysis requirement. The process of analysis describes the static and dynamic characteristics of the system from the aspects of system 
functions, data used by system, the system's behavior process and so on[4]. The mission of requirements analysis is to determine the function of the new system on the basis of investigating the objects to be processed in real-world, fully understanding the work profiles of original system and clearing the various requirements of users. It has the following requirements for the system: functional requirements, performance requirements, reliability and availability requirements, error handling requirements, interface requirements, constraints, reverse demand; may occur in the future requirements. In this paper, using of UML modeling technology to build functional requirements analysis use case diagram for music education resource library, and then describing the function of music education resource library.

Traditional methods of the requirements analysis have three kinds, including process-oriented, information engineering and object-oriented, specific steps are as follows [5]:

(1) Investigating the situation of organization, it includes understanding the composition department of the organization, the functions of departments and so on, to prepare for the analysis of information flow.

(2) Investigating the business activities of various departments, it includes understanding the various departments input and use what data, and how processed the data, what does information output, output to which department, what is the output format.

(3) To help users clear requirements for the new system, including information requirements, processing requirements, completeness and integrity requirements.

(4) Determining the boundaries of the new system, Determine which features completed by the computer or in the future prepared to allow the computer to complete, what activities done by hand. The functions completed by the computer are the functions that should be implemented by the new system.

(5) Analyzing of system functions, system data and preparing the analytical reports.

The specific process of requirement analysis in this paper is: Administrator by logging music education resource library to achieve resource management, resource upload, notice management, user management, message management, system settings, resource statistics and other functions; users through registered and logged on the music education resource library, to search, read and download music education resources, and to collect some excellent resources or to share with others. Use case diagram on music education resources library is shown in Fig. 1.

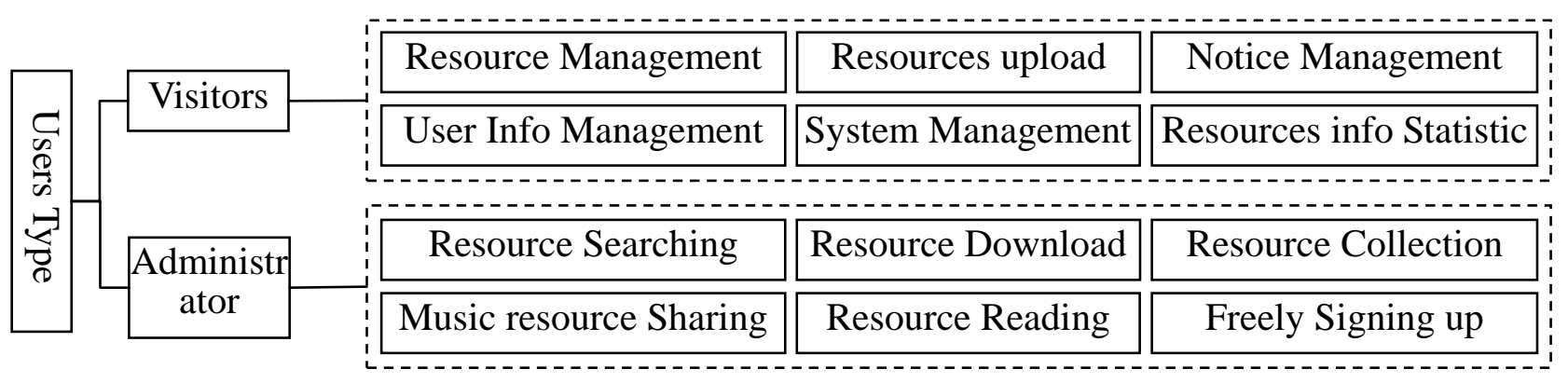

Fig. 1. Functional use case diagram on music education resource library

\section{MVC Framework}

The user interface of (GUI) development and design of music education resources library based on Web studied in this paper uses the MVC design pattern, the full name of MVC is the Model View Controller, is the abbreviations of model - view - controller, a software design model, with a method of business logic, data, interface display separation organize code, the business logic is gathered in a component, improving and customization interface and user interaction, does not need to re-write the business logic. The MVC pattern separates data processing, the input and output control of program and data representation, and describes the communication between the objects in different parts, so that they need not involved in each other's data models and the methods and the 
structure of the program becomes clear flexible [6]. MVC development is mainly used to map the traditional input, processing and output functions in a logical graphical user interface structure.

MVC is a pattern using MVC (Model View Controller) to design and create Web applications: Model represents the application core (such as database records list), is a part of the application for processing application data logic. Usually the model objects responsible for accessing data in the database. View displays data (database records), is a part of the application for processing the data displayed, usually view is created based on the model data. Controller processes the input (write a database record), is a part of the application for processing the user interaction. Typically the controller is responsible for reading data from the view, controlling user input, and transferring data to the model [7].

The design pattern of MVC is shown in Fig. 2.

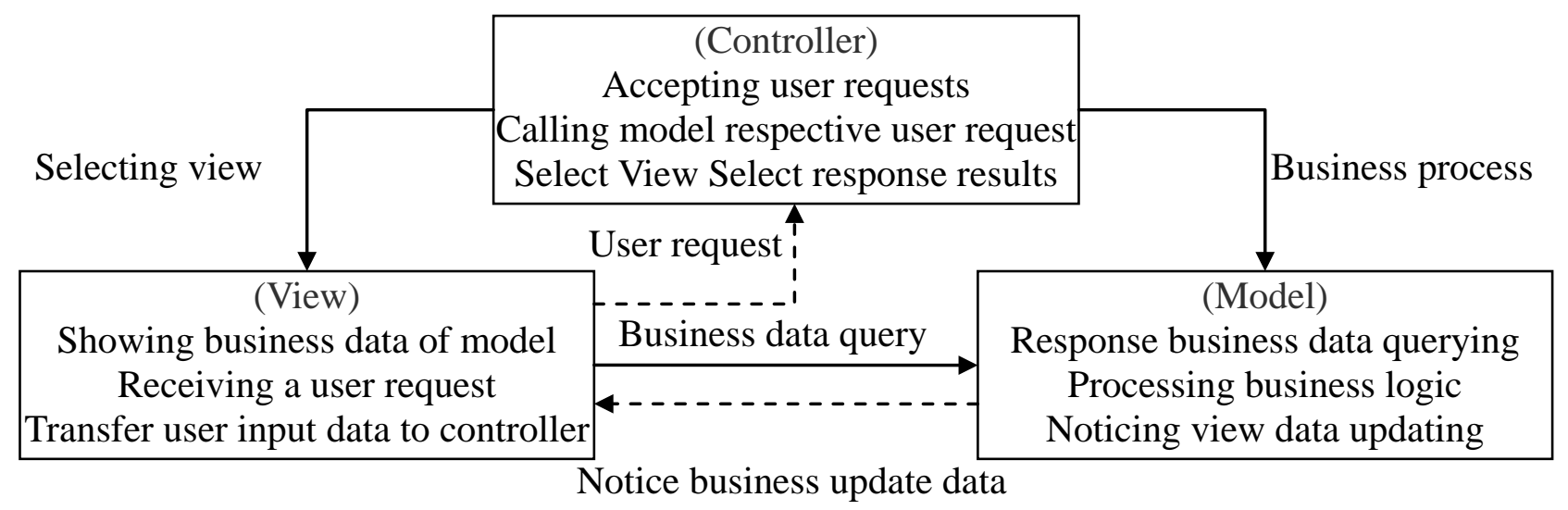

Fig. 2. The design pattern of MVC

\section{Detail Design}

Detail design is a refinement for system architecture designing, mainly based on the requirements specification and architectural design of the system, further refined classes and objects used in the system use case and determined the properties and methods of each class [8]. Analyze detailed features of the system using a structured methodology. In the process of requirements, design and implementation and maintenance for software development, through structural analysis method software functions to be divided, arranging software development time to achieve the goal of tasks division and decomposition. This paper studied the development and design of music education resources library based on Wed; its development design mainly includes reading resources, resource management design, resource retrieval design, system settings, user management design, and resource statistical design and so on.

Resource reading resource primarily achieved arrangement viewing, reading operating authority for resources, specifically including reading music-related information; resource management design includes uploading resources, modify resources, import resources, delete resources, define resources and other methods of review; resource retrieval design defined more methods related to the resource retrieval management, including a query resources, full-text search, keyword search, fuzzy search and synonyms retrieval. System setting is primarily setup issues for music education resources library, including the creation of a packet, and so on; users management design mainly manages user information, including add, find, delete user data; statistical design resources, resource statistical design is mainly embodied in the form of statistical reports and statistics straight graphs.

In which, the design principle in the section of resource retrieval design is information retrieve theory, information retrieval is based on the user's query requirement information to obtain related documentation from a large number of stored documents. Discrimination related documents and irrelevant document information is the key issues for retrieving. Retrieval model needs to implement the appropriate sorting operations of relevant documentation and to select 
model to determine whether a document is mathematical model with relevance, based on the calculation method of similarity degree makes retrieval model develop [9]. Data storage structure directly affects the quality and speed of query, so using the search engine further enhance the retrieval speed of resources, according to its core technology search engine can be divided into two categories: keyword-based search engine and index-based classification search engine. The fundamental difference between them is a different way of building a database [10].

\section{Conclusion}

With the rapid development of computer technology and information technology, the Internet has dramatically changed the way access music for people [11], for the development and design of music education resources library, its design should reflect the comprehensiveness, integrity and openness, openness is to achieve sustainable development of resource development. Although a lot of music researchers plunge into digital music research and rescue and dissemination of music education resources, our musical resources are still facing a severe situation: a large number of music education resources is seriously losing, in the domestic, the platform using for collection, storage, protection, management and sharing music education resources is still very rare, and existing music education resources library has poor scalability, reusability is not high, cannot establish a common and customizable database for music education resource, so we need to combine the appropriate standards, build highly scalable general music education resource library that provides a solid foundation for inheriting a large number of music education resources, standardized managing and creating more standardized music education resource library [12]. music education resource library is designed in this article not only broke the previous features of resource library building just as informative "Save" routine, and promoted services from static service traditions resources to convert to a dynamic service, and became a innovative growth point for the professional and multi-dimensional services, for storing, retrieving, accessing all kinds of music education resources and music development has a great significance.

\section{References}

[1] D. G. Wu, "Thinking on Intangible Cultural Heritage Protection: in the Title of Traditional Music Culture Type," People's Music, vol. 57, no. 1, pp. 34-35, 2006.

[2] C. C. He, "Design and Establishment of Yunnan Minority Music Database," Master's Degree of University of Electronic Science and Technology, 2011.

[3] H. Li, "Discussion on Art Characteristics School Music Library Management," Mass Literature and Art, vol. 56, no. 19, pp. 237, 2011.

[4] Y. L. Chu, "Teaching Resources Management System Research for the Original Ecology Music," Master's Degree of University of Electronic Science and Technology, 2012.

[5] Baidu Baike, "namespace," http://baike.baidu.com/view/111493.htm?fr=aladdin, 2014-9-18.

[6] F. Z. Ren, H. Zhang, S. M. Yan, F. S. Chen, "Overview of the Research in Model - View Controller Pattern," Application Research of Computers, vol. 20, no. 10, pp. 1-4, 2004.

[7] Baidu Baike, "namespace," http://baike.baidu.com/view/5432454.htm?aladdin, 2014-9-18.

[8] Y. D. Yang, "The Design and Implementation of Music education resources Management System for Hunan International Economics University," Master's Degree of Shandong University, 2011.

[9] P. P. Liu, "The Design and Implementation of Retrieval System of Educational Resources Based on XML," Master's Degree of Inner Mongolia University, 2012. 
[10] P. J. Song, C. C. Wang, "Information Storage Technology in Search Engine," Journal of The China Society For Scientific and Technical Information, vol. 20, no. 5, pp. 515-518, 2001.

[11] B. Wang, "Development of Chinese Music under Digital Background," Mass Literature and Art, vol. 55, no. 10, pp. 14, 2010.

[12] J. L. Zhang, "Design and Implementation of Music Database System," Master's Degree of Huazhong University of Science and Technology, 2013. 